\title{
Grounding Grid Fault Diagnosis Based on Empirical Wavelet Transform
}

\author{
Ding Liang ${ }^{1}$, Peng Minfang ${ }^{1}$, Shen Meie ${ }^{2}$, Yan Juan ${ }^{1}$, Ding Xianbing ${ }^{1}$ \\ 1. College of Electrical and Information Engineering, Hunan University, Changsha410000, China \\ 2. College of Computer Science, Beijing University of Information Science and Technology, Beijing \\ 100101, China
}

Keywords: fault diagnosis; grounding grid ; adaptive signal decomposition; empirical wavelet transform; high frequency model

\begin{abstract}
A new adaptive signal processing method based on empirical wavelet transform (EWT) is proposed for the fault diagnosis of grounding grid. According to the frequency domain maximum adaptive point is extracted to split Fourier spectrum to separate the different modes. The establishment of a set of wavelet filter bank is used for partitioned spectrum filtering, extracting components supporting compactly Fourier spectrum AM-FM. In this paper, the high frequency model of grounding grid is built by EMTP simulation software. The method of EWT can analyze the high-frequency signal after grounding grid fault accurately, reveal the frequency structure of fault data effectively and distinguish corrosion severity of grounding grid. It provides a new method for fault diagnosis of grounding grid.
\end{abstract}

\section{Introduction}

Grounding grid is a large hidden works in the power system, with a large number of metal conductor buried deep in the soil. Due to the electrochemical reaction and other factors the conductor will be gradually corroded, the grounding performance becomes poor, even completely by corrosion and loses grounding function[1]. Once grounding grid loses the grounding function, in the abnormal working state of the current, due to the role of heat and power will fracture, the fracture of the grounding grid will bring serious consequences to the safe operation of the power system, resulting in substation equipment damage and personal injury[2]. In order to take the soil discharge around the grounding grid and the interaction between the grounding conductor into account, a high frequency model of grounding grid is established, which can truly reflect the actual operating environment of the grounding grid. Grounding grid high frequency fault signal is generally nonstationary, nonlinear, non Gauss signal, the signal analysis methods are often used in short time Fourier transform, Wigner-Ville distribution, wavelet transform and so on. Once the window function of short time Fourier transform is selected, the time frequency resolution will be fixed[3], does not conform to the actual problem of high frequency signal resolution should be higher than the low frequency signal resolution requirements. Although the Wigner-Ville distribution has very high time and frequency domain resolution, but it is limited by the existence of cross interference[4]. Although the wavelet transform has the characteristics of multi scale, it can be observed by the coarse and fine gradually, but because of the need for artificial selection of wavelet basis, so self adaptability is lacked[5].

Empirical wavelet transform (EWT) is a new signal processing method proposed by Gilles[6], based on the adaptive division of the signal, a set of wavelet filter banks is established to filter the spectrum, the instantaneous frequency and amplitude can be obtained by using the Hilbert transform of each single component component[7-8].

By building the high frequency model of the grounding grid, EWT can accurately analyze the high frequency fault signal, and the fault feature is obvious, which can be effectively applied to the fault diagnosis of grounding grid. 


\section{Empirical Wavelet Transform}

Empirical wavelet transform is actually based on the theoretical framework of wavelet analysis , according to the characteristics of the signal spectrum a set of wavelet filters are selected. In order to determine the frequency range of the filter, the Fourier spectrum of the signal is divided. Assuming that Fourier supporting $[0, \pi]$ is divided into $\mathrm{N}$ continuous parts, $\omega_{n}$ is used to represent the boundary between the segments, $\omega_{n}$ is selected as the midpoint between the two adjacent maximum points of the signal Fourier spectrum, each segment can be expressed as:

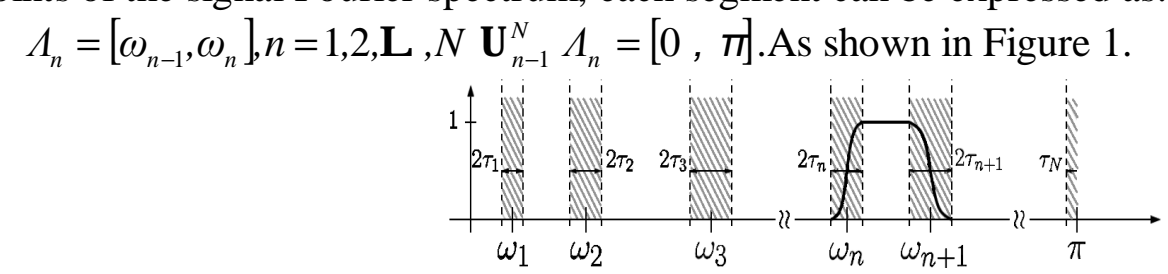

Fig.1 Partitioning of the Fourier axis

Figure in the transition region to $\omega_{n}$ as the center, the width of $\tau_{n}$. According to determine the partition interval $\Lambda_{n}$, empirical wavelet is defined as a band-pass filter on each $\Lambda_{n}$. According to the construction method of Meyer wavelet[9], Gilles constructs empirical wavelet, empirical wavelet function $\hat{\psi}_{n}(\omega)$ and empirical scaling function $\hat{\varphi}_{n}(\omega)$ are shown in Formula 1 and 2 respectively.

$$
\begin{gathered}
\hat{\varphi}_{n}(\omega)=\left\{\begin{array}{cc}
1 & \text { if }|\omega| \leq \omega_{n}-\tau_{n} \\
\cos \left[\frac{\pi}{2} \beta\left(\frac{1}{2 \tau_{n}}|\omega|-\omega_{n}+\tau_{n}\right)\right] \\
\text { if } \omega_{n}-\tau_{n} \leq|\omega| \leq \omega_{n}+\tau_{n} \\
0 \quad & \text { otherwise }
\end{array}\right. \\
\hat{\psi}_{n}(\omega)=\left\{\begin{array}{cc}
1 & \text { if } \omega_{n}+\tau_{n} \leq|\omega| \leq \omega_{n+1}-\tau_{n+1} \\
\cos \left[\frac{\pi}{2} \beta\left(\frac{1}{2 \tau_{n+1}}\left(|\omega|-\omega_{n+1}+\tau_{n+1}\right)\right)\right] & \text { if } \omega_{n+1}-\tau_{n+1} \leq|\omega| \leq \omega_{n+1}+\tau_{n+1} \\
\sin \left[\frac{\pi}{2} \beta\left(\frac{1}{2 \tau_{n}}|\omega|-\omega_{n}+\tau_{n}\right)\right] \\
\text { if } \omega_{n}-\tau_{n} \leq|\omega| \leq \omega_{n}+\tau_{n} \\
0 \quad \text { otherwise }
\end{array}\right.
\end{gathered}
$$

Formula:

$$
\begin{aligned}
& \beta(x)=x^{4}\left(35-84 x+70 x^{2}-20 x^{3}\right) \\
& \tau_{n}=\gamma \omega_{n} \\
& \gamma<\min _{n}\left(\omega_{n+1}-\omega_{n} / \omega_{n+1}+\omega_{n}\right)
\end{aligned}
$$

In the EWT method, the segmentation of Fourier spectrum is directly related to the result of adaptive decomposition. The different frequency spectrum corresponds to the mode of the center of the spectrum with different specific tight support.Remove the 0 and $\pi$, also need to find a N-1 boundary.According to the construction method of the classical wavelet transform, the empirical wavelet transform is constructed, and the detail coefficients and the approximation coefficients are respectively[10]:

$$
\begin{aligned}
& \omega_{f}^{\varepsilon}(n, t)=\left\langle f, \psi_{n}\right\rangle=\int f(\tau) \overline{\psi_{n}(\tau-t)} d \tau= \\
& \left(\hat{f}(\omega) \overline{\hat{\psi}_{n}(\omega)}\right) \vee
\end{aligned}
$$




$$
\begin{aligned}
& \omega_{f}^{\varepsilon}(0, t)=\left\langle f, \varphi_{1}\right\rangle=\int f(\tau) \overline{\varphi_{1}(\tau-t)} d \tau= \\
& \left(\hat{f}(\omega) \overline{\hat{\varphi}_{1}(\omega)}\right) \vee
\end{aligned}
$$

Signal reconstruction, as shown in the formula (8):

$$
\begin{aligned}
& f(t)=\omega_{f}^{\varepsilon}(0, t) \times \phi_{1}(t)+\sum_{n=1}^{N} \omega_{f}^{\varepsilon}(n, t) \times \psi_{n}(t)= \\
& \left(\hat{\omega}_{f}^{\varepsilon}(0, \omega) \hat{\phi_{1}}(\omega)+\sum_{n=1}^{N} \hat{\omega}_{f}^{\varepsilon}(n, \omega) \hat{\phi}_{n}(\omega)^{\vee}\right.
\end{aligned}
$$

By the above formula to obtain the empirical mode function $f_{k}$, can be expressed by the formula (9) and the formula (10).

$$
\begin{gathered}
f_{0}(t)=\omega_{f}^{\varepsilon}(0, t) \times \varphi_{1}(t) \\
f_{k}(t)=\omega_{f}^{\varepsilon}(k, t) \times \psi_{k}(t)
\end{gathered}
$$

Through the empirical wavelet transform, we can get the empirical mode of a signal, and we can carry out Hilbert transformation to each empirical mode function, and then the instantaneous frequency and instantaneous amplitude can be obtained, which can accurately describe the change of the frequency time.

\section{High frequency excitation model}

In order to reflect the actual situation of the grounding grid,we consider the high frequency AC excitation, taking into account the inductance of the grounding body as well as the capacitance effect between the conductor and the soil,establish the grounding grid model under the action of high frequency excitation.Under high frequency excitation, the grounding grid will appear and the low frequency is not the same, the inductance effect will be better reflected, which provides more information for the fault diagnosis of grounding grid. One of the electrical parameters of any ground branch conductor includes a resistance $\mathrm{R}$, an inductance $\mathrm{L}$, a ground conductor $\mathrm{G}$ and a ground capacitors $C$, the resistance $\mathrm{R}_{0}$, capacitance $\mathrm{C}_{0}$, inductance $\mathrm{L}_{0}$, and capacitance $\mathrm{C}_{0}$ of the unit length can be expressed[11-12].

$$
\begin{aligned}
& G=\frac{\pi l}{\rho(\ln (2 l / \sqrt{2 r h}-1))} \\
& C=\varepsilon_{0} \varepsilon_{r} \rho G \\
& L=\frac{\mu_{0} l}{2 \pi\left(\ln \frac{2 l}{r}-1\right)}
\end{aligned}
$$

Among them, $l$ is the length of the earth electrode; $h$ is the grounding depth; $r$ is the equivalent radius of the earth electrode; $\varepsilon_{r}$ is the relative dielectric constant of the soil; $\varepsilon_{0}$ is the relative medium; $\rho$ is the soil resistivity; $\mu_{0}$ is the magnetic coefficient of the soil. In EMTP software, we replace the equivalent unit structure of each branch grounding conductors by $\pi$ equivalent circuit element, equivalent circuit as shown in Figure 2.

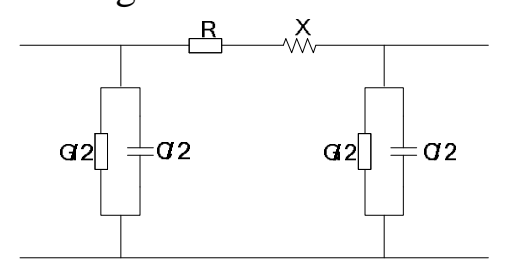

Fig. $2 \pi$-style equivalent circuit

Taking the different frequency of signal attenuation in the soil into account, the frequency range of the high-frequency model established in this paper is $10 \mathrm{kHz}-100 \mathrm{kHz}$, the greater the frequency 
of the excitation source, the inductive component is more obvious.

\section{Grounding grid simulation research}

The EMTP software is used to build the $6 \times 6$ simulation circuit of the grounding grid as shown in Figure 3.The frequency of the voltage excitation is $60 \mathrm{KHz}$, the amplitude is $200 \mathrm{~V}$. In order to reduce the influence of the position of the excitation source on the result, the oblique excitation method as shown in figure 3 is adopted.The number of nodes from top to bottom, from left to right in order to represent the number of Arabia. When a branch is at fault, such as the occurrence of corrosion, which leads to the branch of capacitance increases, through changing each branch conductor of the parameters can be simulated accurately ground grid corrosion, such as selecting nodes $(9,10)$ as the detection nodes.

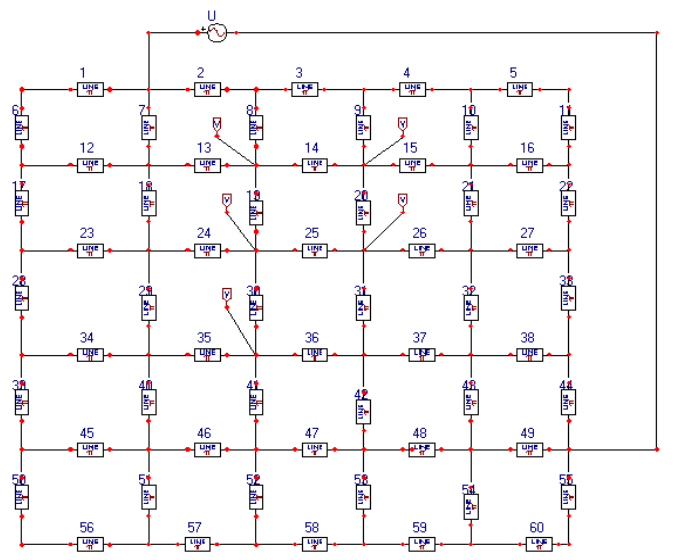

Fig. 3 Simulation circuit of grounding grid

When the branch 14 is normal, slight corrosion and severe corrosion occurs, the voltage response of the node in different state is shown in Figure 4.

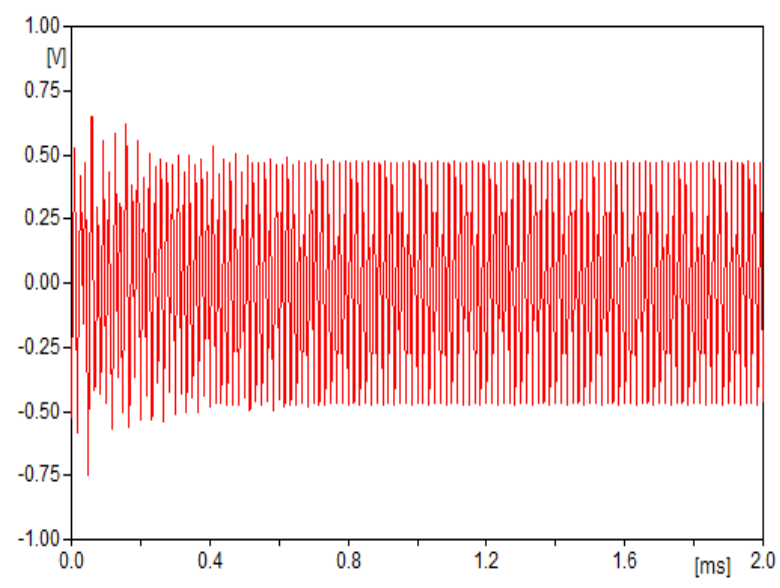

(a)no corrosion

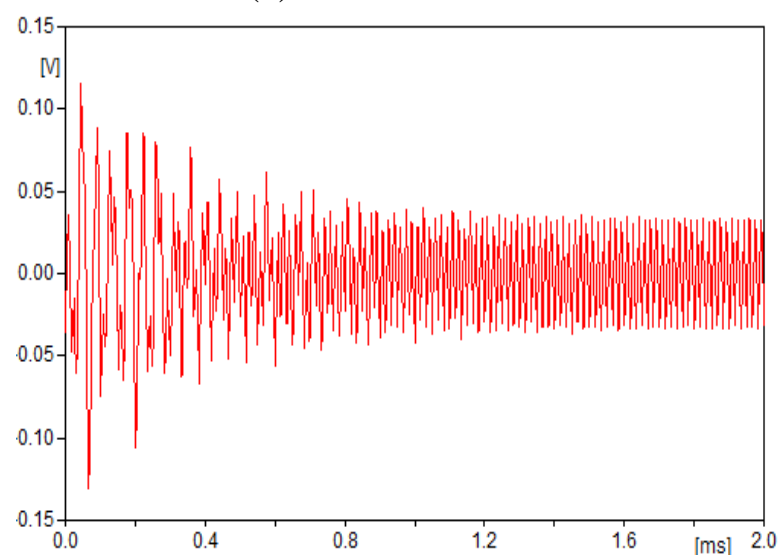

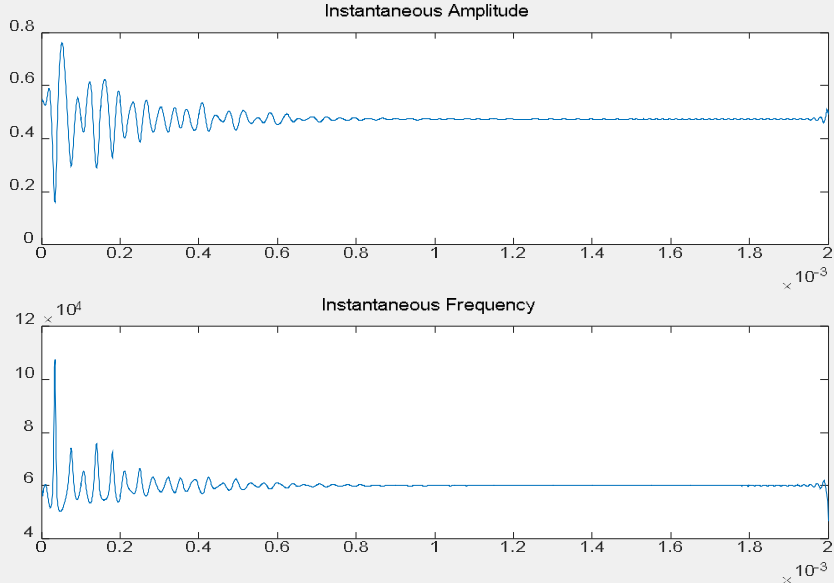

(a) no corrosion
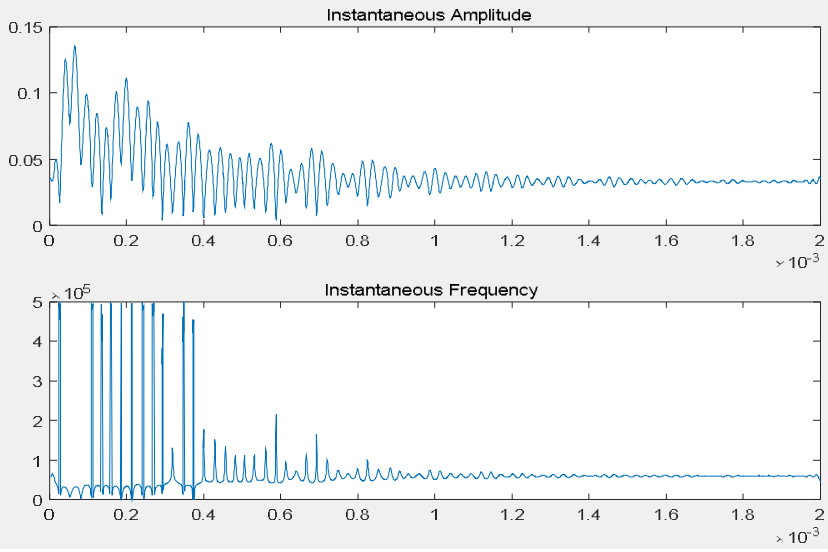
(b) slight corrosion

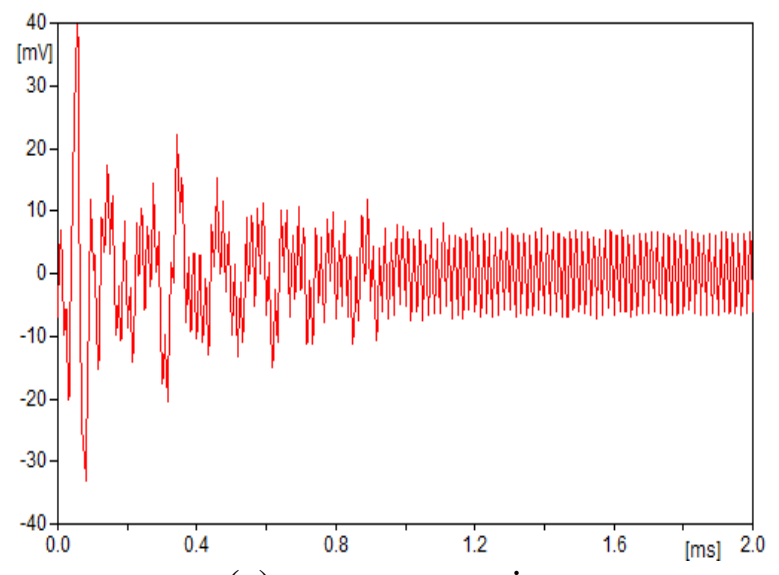

(c) severe corrosion

Fig. 4 Detection node voltage response diagram (b) slight corrosion
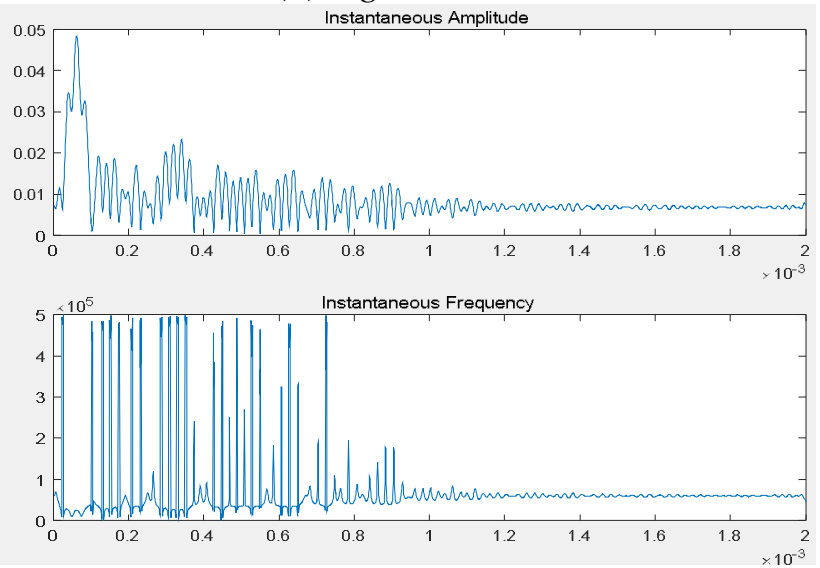

(c) severe corrosion

Fig. 5 Instantaneous amplitude and frequency of node voltage

Through the empirical wavelet transform. By the detection of node voltage respon sediagram instantaneous amplitude and instantaneous frequency detection node voltage response, as shown in Figure 5.

From Figure 5 (a) can be known: grounding grid corrosion phenomenon is not occurred, and the $60 \mathrm{KHz}$ frequency of the excitation source is compared, the instantaneous frequency of the signal is shown in addition to the $1 \mathrm{X}$ frequency, there is a frequency of $1 \mathrm{X}$ to $2 \mathrm{X}$, and the instantaneous amplitude of the voltage response is larger. From Figure 5 (b) and (c) can be known:When corrosion occurs, the frequency component of the signal appears to be very rich, in addition to $1 \mathrm{X}$ frequency, also appeared higher order frequency, such as: $6 \mathrm{X}, 7 \mathrm{X}$ frequency doubling, and the number of higher order frequency increases with the increase of the degree of corrosion. When the grounding grid corrosion, the instantaneous amplitude and frequency of the signal changes with time and finally tends to be stable, the more serious corrosion, the longer the time to stabilize.

Through the above analysis, it is known that the EWT transform can effectively decompose the instantaneous amplitude and frequency of the grounding grid corrosion fault signal with time. When the grounding grid is not corroded, the low order frequency exists, and the high order frequency component is not completely. With the occurrence of corrosion, the low order frequency still exists, the higher order of the frequency components with the increase of the degree of corrosion more and more, and the instantaneous amplitude decreased significantly.

\section{Conclusion}

The empirical wavelet transform (EWT) through the spectrum of the signal is divided adaptively, constructing an appropriate orthogonal wavelet filter banks to extract AM-FM components with compactly supported Fourier spectrum, and then the Hilbert transform, instantaneous frequency and amplitude of the meaningful.In this paper, the EWT method is introduced in detail, and the method is referenced to the grounding grid corrosion diagnosis. The fault diagnosis method based on empirical wavelet decomposition is proposed, and the simulation experiment is carried out.The simulation results show that the proposed method can effective decompose the fault signal with the time variation of the instantaneous amplitude and frequency, which can be intuitive judgment that the corrosion status of grounding grid and provides a new approach to fault diagnosis of grounding grid.

\section{Acknowledgements}

This work is supported by National Natural Science Foundation of China under Grant No.61472128 and 61173108, and Hunan Provincial Natural Science Foundation of China No.4JJ2150. 


\section{References}

[1] Zhu Zhiping, Xiao Ma, Jing Lingling. Soil corrosion evaluation of substation and grounding grid corrosion characteristics analysis [J]. Insulators and Surge Arresters, 2009 (4):18-22

[2] Yang Daowu, Chen Kunhan. Evaluation of soil corrosion resistance of electric grounding grids [J]. Corrosion and Protection, 2008,29 (10):614-617

[3] Zhang Ye. Signal time-frequency analysis and application [M]. Harbin: Harbin Institute of Technology, 2006

[4] XIANG Ling ,TANG Guiji,HU Aijun,Vibration signals time-frequency analysis and comparison for a rotating machinery[J].Journal of Vibration and Shock,2010,29(2):42-45

[5] YEH Poliang,LIU Peiling.Application of the wavelet transform and the enhanced Fourier spectrum in the impact echo test[J].NDT and E International,2008,41(5):383-394.

[6] GILLES J.Empirical wavelet transform[J].IEEE Transactions on Signals Processing, 2013,61(16):3999-4010.

[7] Li Zhinong, Zhu Ming. Mechanical fault diagnosis method based on empirical wavelet transform [J].Chinese Journal of Scientific Instrument,2014,35 ( 11 ) : 2423-2431

[8] Xiang Ling, Li Yuanyuan. Application of empirical wavelet transform in fault diagnosis of rotating machinery [J]. Chinese Journal of Power Engineering, 2015,35 (12):975-981

[9] DAUBECHIES I.Ten lectures on wavelets [M].Philade- lphia:Society for Industrial and Applied Mathematics, 1992.

[10] FRANCIS A,MURUGANANTHAM C.An adaptive denoising method using empirical wavelet transform[J].International Journal of Computer Applications,2015,117(21):18-20

[11] Xiang Ling, Hu Minqiang, Zheng Jianyong.EMTP in lightning simulation of substations grounding grid[J].High Voltage Engineering,2005,31(6):69-72.

[12] Li Xiaofang, Peng Minfang, Luo Luo. Information fusion fault diagnosis of substation grounding grid [J]. Proceedings of the CSU-EPSA, 2015,12 (27):36-41. 